\title{
A Flux-Pinning Mechanism for Segment Assembly and Alignment
}

\author{
Jessica A. Gersh-Range ${ }^{\mathrm{a}}$, William R. Arnold ${ }^{\mathrm{b}}$, Mason A. Peck ${ }^{\mathrm{a}}$, H. Philip Stahl ${ }^{\mathrm{c}}$ \\ ${ }^{a}$ Cornell University, Ithaca, NY USA 14850; ${ }^{b}$ Jacobs ESTS Group, Marshall Space Flight Center, \\ Huntsville, AL USA 35812; ${ }^{\circ}$ NASA, Marshall Space Flight Center, Huntsville, AL USA 35812
}

\begin{abstract}
Currently, the most compelling astrophysics questions include how planets and the first stars formed and whether there are protostellar disks that contain large organic molecules. Although answering these questions requires space telescopes with apertures of at least 10 meters, such large primaries are challenging to construct by scaling up previous designs; the limited capacity of a launch vehicle bounds the maximum diameter of a monolithic primary, and beyond a certain size, deployable telescopes cannot fit in current launch vehicle fairings. One potential solution is connecting the primary mirror segments edgewise using flux-pinning mechanisms, which are analogous to non-contacting damped springs. In the baseline design, a flux-pinning mechanism consists of a magnet and a superconductor separated by a predetermined gap, with the damping adjusted by placing aluminum near the interface. Since flux pinning is possible only when the superconductor is cooled below a critical temperature, flux-pinning mechanisms are uniquely suited for cryogenic space telescopes. By placing these mechanisms along the edges of the mirror segments, a primary can be built up over time. Since flux pinning requires no mechanical deployments, the assembly process could be robotic or use some other noncontacting scheme. Advantages of this approach include scalability and passive stability.
\end{abstract}

Keywords: segmented mirror, space telescope, flux pinning, cryogenic

\section{INTRODUCTION}

Currently, some of the most compelling astrophysics questions concern the origin and evolution of the universe and the prevalence of life, and the desire to answer these questions creates a need for large-aperture space telescopes that complement existing observatories. A 10- to 16-m class far-infrared to submillimeter space telescope, for example, is required to answer questions about early star formation, planetary system evolution, and the presence of large organic molecules in protostellar disks. These measurements cannot be performed with any existing observatory since atmospheric extinction precludes ground-based measurements at these wavelengths and since the space telescopes that operate in this range suffer from source confusion. ${ }^{1-4}$

While science drivers exist for creating large-aperture space telescopes, such large primaries are challenging to package and deploy. Since the limited capacity of a launch vehicle places an upper bound on the diameter of a monolithic mirror, larger apertures require segmentation, which can be implemented using a variety of methods. The first segmented space telescope, the James Webb Space Telescope, has a primary consisting of 18 hexagonal segments mounted on a rigid backplane that folds to meet launch fairing constraints, ${ }^{5}$ and some proposals for even larger segmented telescopes use a scaled version of this design., ${ }^{3,6,7}$ Alternative proposals rely on tethers to connect the segments, with rotation providing the stability instead of a backplane. ${ }^{8,9}$ However, while these techniques seek to maximize the diameter given the launch fairing constraints, an upper limit remains. Achieving even larger diameters requires decoupling the mirror packaging and deployment scheme from the choice of launch vehicle, which can be accomplished by launching a collection of segments that are assembled in space. Assembly methods include formation flight, ${ }^{10}$ electromagnetic formation flight, ${ }^{11}$ and laser trapping. ${ }^{12,13}$ Yet, these techniques present their own challenges. They require active control to prevent collisions and maintain optical alignment, and some techniques, such as formation flight, require the use of expendables. Ideally, the assembly method would enable the on-orbit construction of a large segmented primary that does not rely on active control to maintain its shape.

Flux-pinning mechanisms may provide a means of attaining this ideal scenario. A non-contacting interaction between a magnet and a type II superconductor, flux pinning passively stabilizes the relative orientation and position of the magnet and superconductor in every direction that has a magnetic field gradient. A more detailed description of flux 
pinning is presented in Section 2. A flux-pinning mechanism consists of an arrangement of at least one magnet and one superconductor, and like a flexure, it allows free motion in specific degrees of freedom. In any other degree of freedom, there is a restoring force analogous to a damped spring force. Section 3 describes sample mechanisms and their properties. Since flux pinning requires cryogenic temperatures, a flux-pinning mechanism is activated only once the superconductor cools below some critical temperature. As a result, these mechanisms can be used to connect adjacent mirror segments in a non-contacting manner on-orbit, assuming that there exists some mechanism to maintain at least coarse alignment while the segments cool down. The aim of this approach is to assemble a segmented mirror that is analogous to a monolith; the segments are physically connected to their neighbors at discrete locations, rather than floating in a formation that must be actively controlled. Section 4 presents potential mirror assembly schemes, and Section 5 summarizes the mechanism concept and its proposed application.

\section{FLUX PINNING}

A type of interaction between a magnet and a type II superconductor, flux pinning is analogous to a damped spring force that acts over a distance and is due to the presence of impurities in the superconductor material. These impurities allow magnetic fields to penetrate into the superconductor; if the superconductor is cooled below some critical temperature $T_{c}$ (88K for YBCO, yttrium barium copper oxide) in the presence of a magnetic field, the magnetic field lines are "frozen" into the superconductor, and any subsequent changes to the magnetic field induce supercurrents that oppose the change. As a result, the relative orientation and position of the magnet and superconductor are passively stabilized in every direction that has a magnetic field gradient.

In the frozen-image model, ${ }^{14}$ the interaction between the permanent magnet and the superconductor can be explained by using three magnets, as shown in Figure 1. The first magnet corresponds to the permanent magnet, and the remaining magnets are images of the permanent magnet within the superconductor. The first image magnet, the mobile image, is a reflection of the permanent magnet across the superconductor surface. The mobile image moves as the permanent magnet moves, and since their dipole moments are mirror images, the two magnets always repel. The second image magnet, the frozen image, is almost a reflection of the permanent magnet at the moment the superconductor cooled below its critical temperature; the orientation and position are mirror-imaged, but the dipole moment is the same. The frozen image does not change as the permanent magnet moves. The force on the permanent magnet is equal to the sum of the forces of the two image magnets, and the orientation and position the permanent magnet has as the superconductor cools below its critical temperature define an equilibrium orientation and position.

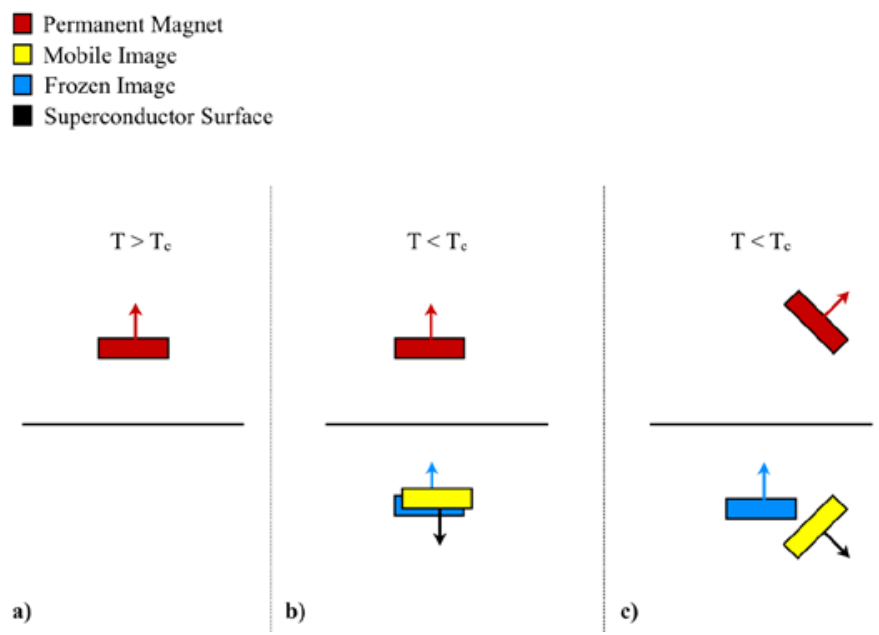

Figure 1. The frozen-image model.

In the frozen-image model, the interaction between a magnet and a type II superconductor is represented by the interaction between the magnet and two image magnets: a mobile image magnet, which is a mirror image of the permanent magnet, and a frozen image magnet, which is a mirror image of the permanent magnet at the moment the superconductor cools below its critical temperature with the dipole moment reversed. When the superconductor temperature is above the critical temperature, as shown in a), there is no interaction between the magnet and the superconductor. The image magnets form once the superconductor cools below its critical temperature, as shown in b). If the permanent magnet later moves away from its initial position, the mobile image follows, while the frozen image remains fixed, as shown in c). 


\section{THE FLUX-PINNING MECHANISM}

A flux-pinning mechanism consists of a configuration of at least one magnet and one superconductor, with the specific arrangement determined by the mechanism application. Previous investigations into flux-pinning mechanism design have focused on the application of these mechanisms as joints between spacecraft modules, ${ }^{15,16}$ but the same principles apply to designing optomechanical devices. Like a flexure, a flux-pinning mechanism preferentially allows motion in specific degrees of freedom, and the choice of these degrees of freedom affects how the mechanism is constructed.

For example, to allow motion in five degrees of freedom like a wire flexure, a flux-pinning mechanism could consist of a superconductor pinned to a spherical magnet mounted in an inverted cone, as shown in Figure 2a. Since the line connecting the spherical magnet and the superconductor is an axis of symmetry for the magnetic field, the superconductor is free to rotate about this axis without disturbing the magnet. However, a spring-like restoring force will oppose any motions that would change the relative orientation and position of the magnet and superconductor, such as motion along the line connecting the two. As a result, rotating the superconductor about the cone will cause the magnet to rotate correspondingly since it is free to move within the cone.

To allow motion along a line like a pair of parallel blade flexures, a flux-pinning mechanism could consist of a superconductor pinned between two long cylindrical magnets, as shown in Figure 2b. ${ }^{*}$ In this configuration, a line between and parallel to the magnets is the only direction without a magnetic field gradient, so it is the only direction in which the superconductor can move freely. To allow only rotation about an axis like a cross-blade flexure, a fluxpinning mechanism could consist of a small cylindrical magnet pinned above a superconducting disk, as shown in Figure 2c. As with the mechanism in 2a, the line connecting the magnet and superconductor is an axis of symmetry for the magnetic field, so rotations about this axis are unconstrained. Motions in any other direction, however, are opposed by a restoring force since there is a magnetic field gradient and the entire mechanism is not free to rotate. In each of these examples, the flux-pinning mechanism design is driven by the degrees of freedom in which motion is desired. Shaping the magnetic field to be constant in all of these directions is one approach but not a requirement, as the mechanism in $2 \mathrm{a}$ demonstrates.

\footnotetext{
* This configuration corresponds to the prismatic joint described in (Shoer and Peck, 2009).
} 


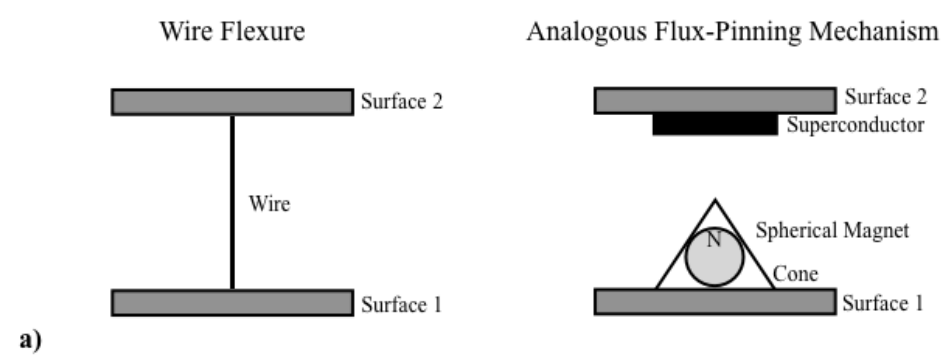

a)

Parallel Blade Flexures

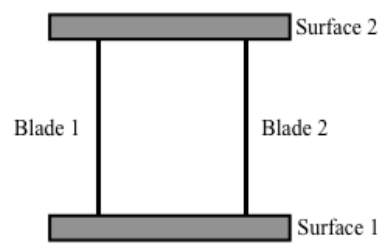

Analogous Flux-Pinning Mechanism

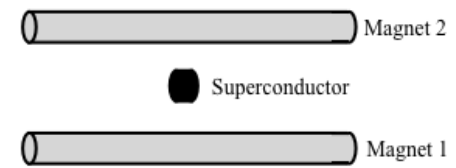

b)

Cross-blade Flexure

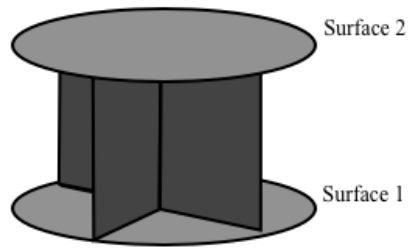

Analogous Flux-Pinning Mechanism

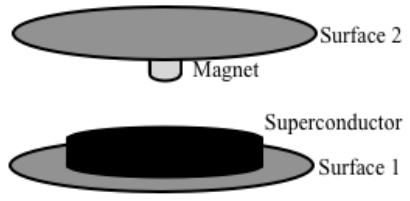

c)

Figure 2. Flexures and the analogous flux-pinning mechanisms.

Like a flexure, a flux-pinning mechanism preferentially allows motion in certain directions, and the chosen directions determine the mechanism design. For example, to allow motion in five degrees of freedom like the wire flexure in a), the flux-pinning mechanism consists of a superconductor pinned to a spherical magnet which is mounted inside an inverted cone. To allow motion along a line like the pair of parallel blade flexures in b), the flux-pinning mechanism consists of a superconductor pinned between two long cylindrical magnets. To allow only rotary motion like the cross-blade flexure in c), the flux-pinning mechanism consists of a small cylindrical magnet pinned to a superconducting disk.

The restoring force that opposes motion in directions with a magnetic field gradient is analogous to a damped spring force. The stiffness and damping of this force depend upon the choice of magnet and superconductor, the initial separation between the magnet and superconductor, and the presence of conductors. For a given magnet/superconductor pair, the stiffness of the interaction is determined by the initial separation between the magnet and the superconductor, increasing nearly exponentially as the separation decreases, ${ }^{17}$ and the amount of damping can be adjusted independently by placing nonmagnetic conductive metals, such as aluminum, near the interface. ${ }^{15}$

\section{ASSEMBLY CONCEPTS}

By connecting neighboring segments edgewise, flux-pinning mechanisms may enable the on-orbit assembly of large segmented primaries that are analogous to monoliths. A large segmented primary could, for example, consist of rings of hexagonal segments or petals, with the flux-pinning mechanisms placed along the segment edges, as shown in Figure 3. 


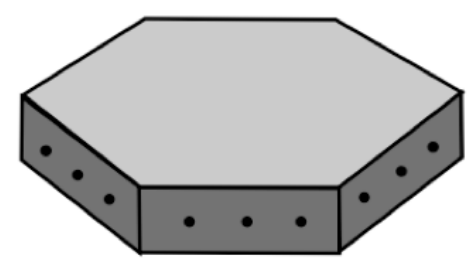

a)

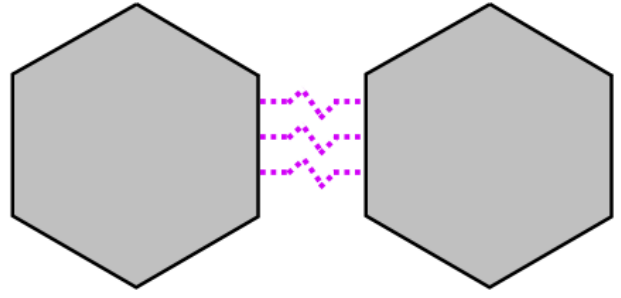

b)

Figure 3. Segments with flux-pinning mechanisms.

The hexagonal segment in a) is an example of a mirror segment with three flux-pinning mechanisms (represented by dots) along each edge. In b) two such segments are flux-pinned together. The dashed lines represent the non-contacting flux-

pinning interaction.

Like a monolithic mirror, such a segmented mirror could be treated as a single unit, although the connections between the segments are discrete instead of continuous. In addition, relative motion between the segments would introduce an amount of damping that can be adjusted by placing nonmagnetic conductive materials, such as aluminum, near the fluxpinning mechanisms. Previous experiments ${ }^{15}$ have demonstrated that the amount of damping can be as high as $2 \%$ of critical, compared to the $0.001 \%$ (or less) material damping typically present in space telescopes. ${ }^{18}$

Since flux pinning is possible only when the superconductor is cooled below a critical temperature, flux-pinning mechanisms are uniquely suited for telescopes that must be kept cold in order to operate, such as infrared telescopes. These non-contact mechanisms operate best at cryogenic temperatures, unlike mechanical mechanisms, which can have problems with lubrication, CTE matching, and thermal snap. In addition, it is possible to deactivate a flux-pinning mechanism and reactivate it in a new configuration simply by warming the superconductor, changing the relative orientation and position of the magnet and superconductor, and allowing the superconductor to cool. However, the operational temperature requirement for flux-pinning mechanisms has another implication: in order to use flux-pinning mechanisms for mirror alignment, some other technique must be used to maintain the alignment as the superconductors cool. In other words, the mirror assembly process would require multiple steps. Three potential assembly schemes are outlined below.

\subsection{Robotic Alignment}

One approach is to use a robotic system to align the flux-pinning mechanisms during the cooling process; once cold, the flux-pinning mechanisms maintain the alignment and phasing. The alignment process consists of three stages. In the first stage, robotic arms coarsely align the flux-pinning mechanisms. In the second stage, other actuators, such as hexapods similar to the ones used on JWST, ${ }^{5}$ finely align the mechanisms. In the final stage, the mechanisms cool below the critical temperature, and the other actuators are powered down.

A variation of this approach uses electromagnets to provide the fine alignment. As before, robotic arms coarsely align the flux-pinning mechanisms. Then, in the second stage, the mechanisms cool below the critical temperature and are finely aligned by electromagnets, which adjust the relative orientation and position of the magnets and superconductors by perturbing the magnetic field. This layer of active control is temporary; to achieve a passive aligned mirror, the mechanisms are reset one at a time. A mechanism is warmed until deactivated, and the electromagnets responsible for controlling its alignment are turned off. The mechanism is allowed to cool, pinning it in the new configuration, and the process is repeated for the remaining mechanisms.

\subsection{Retractable Latches}

Another approach is to assemble the warm mirror by latching the segments together. These latches maintain the alignment as the mirrors cool, then release once the flux-pinning mechanisms are activated. This approach has the advantage of providing a means of maintaining the alignment that requires no power in the event that the mirror must be warmed, such as for servicing; the segments simply latch together again. 


\subsection{Temporary Active Control}

A third approach is to use electromagnetic formation flight ${ }^{11}$ to maintain the alignment as the superconductors cool. These electromagnets are placed sufficiently far away from the flux-pinning mechanisms to prevent any interference, and they are turned off once the flux-pinning mechanisms are activated.

\section{SUMMARY}

A flux-pinning mechanism consists of a configuration of magnets and superconductors that allows unconstrained motion in specific degrees of freedom. Motion in any other degree of freedom gives rise to a restoring force analogous to a non-contacting damped spring force, and the stiffness and damping are mechanism properties that can be adjusted independently. Since flux pinning currently requires cryogenic temperatures, the relative orientation and position of the magnet and superconductor must be maintained by some other means while the superconductor cools. Potential methods include robotic assembly, the use of retractable latches, or electromagnetic formation flight. However, unlike mechanical mechanisms, flux-pinning mechanisms operate best at low temperatures and are ideally suited for use in infrared space telescopes. One potential application of these mechanisms is the assembly and alignment of large segmented cryogenic mirrors; placing these mechanisms along the segment edges may enable the on-orbit assembly of a segmented mirror that is analogous to a monolithic mirror in that it can be treated as a single unit rather than an actively controlled formation. Potential benefits of this approach include passive stability, reconfigurability, and ease of repair.

The successful implementation of a flux-pinning mechanism in an optomechanical application depends largely on the achievable stiffness and damping. While previous work has demonstrated the use of a flux-pinning mechanism for aligning CubeSats, the mechanism was not sufficiently stiff for optical alignment; the CubeSats were separated by a distance on the order of a couple centimeters, which resulted in a stiffness on the order of a few newtons per meter. ${ }^{16}$ However, higher stiffnesses can be achieved by flux pinning the magnet and superconductor closer together or using a stronger magnet. In particular, correlated magnets, which can have a near field that is at least $50 \%$ stronger than that of an equivalent neodymium magnet, may provide a means of achieving a sufficient stiffness. ${ }^{19}$ An investigation into the stiffness and damping of correlated and conventional magnets for separations of at most $1 \mathrm{~mm}$ is in progress and will form the topic of a future paper.

\section{ACKNOWLEDGEMENT}

We would like to thank the NASA Graduate Student Researchers Program for its support of this project.

\section{REFERENCES}

[1] Leisawitz, D., “NASA's far-IR/submillimeter roadmap missions: SAFIR and SPECS,” Advances in Space Research 34 (3), 631-636 (2004).

[2] Thronson, H. A., Postman, M., Stahl, H. P., and Traub, W., “Astronomy enabled by Ares $V$ heavy lift,” Future InSpace Operations White Paper (2007).

[3] Benford, D. J., Amato, M. J., Mather, J. C., Moseley Jr., S. H., and Leisawitz, D. T., "Mission concept for the single aperture far-infrared (SAFIR) observatory,” Astrophysics and Space Science 294, 177-212 (2004).

[4] Rieke, G. H., Benford, D. J., Harvey, P. M., Lawrence, C. R., Leisawitz, D. T., Lester, D. F., Mather, J. C., Stacey, G. J., Werner, M. W., and Yorke, H. W., "Charting the winds that change the universe, II: The single aperture farinfrared observatory (SAFIR)," Proceedings of the Second Workshop on New Concepts for Far-Infrared and Submillimeter Space Astronomy, 157-166 (2002).

[5] Gardner, J. P., Mather, J. C., Clampin, M., Doyon, R., Greenhouse, M. A., Hammel, H. B., Hutchings, J. B., Jakobsen, P., Lilly, S. J., Long, K. S., Lunine, J. I., McCaughrean, M. J., Mountain, M., Nella, J., Rieke, G. H., 
Rieke, M. J., Rix, H.-W., Smith, E. P., Sonneborn, G., Stiavelli, M., Stockman, H. S., Windhorst, R. A., and Wright, G. S., “The James Webb Space Telescope,” Space Science Reviews 123 (4), 485-606 (2006).

[6] Amato, M. J., Benford, D. J., Moseley, S. H., and Roman, J. A., "An engineering concept and enabling technologies for a large single aperture far-infrared observatory (SAFIR),” Proc. SPIE 4850, 1120-1131 (2003).

[7] Postman, M., et al, "Advanced technology large-aperture space telescope (ATLAST): A technology roadmap for the next decade,”arXiv:0904.0941v2 [astro-ph.IM], (2009).

[8] Farley, R. E. and Quinn, D. A., "Tethered formation configurations: Meeting the scientific objectives of large aperture and interferometric science,” AIAA Space 2001 - Conference and Exposition, (2001).

[9] Lorenzini, E., Bombardelli, C., Cosmo, M., Harwit, M., Leisawitz, D., Farley, R., Rinehart, S., Quinn, D., and Miller, D., "Far-infrared/submillimeter astronomical interferometry with spaceborne tether formations," Astrophysics and Space Science 302, 225-239 (2006).

[10] Lawson, P. R., Lay, O. P., Johnston, K. J., Beichmann, C. A., Unwin, S. C., Martin, S. R., Hunyadi, S. L., Absil, O., Akeson, R. L., Bally, J., Bordé, P., Crisp, D., Danchi, W. C., Defrère, D., Eiroa, C., Falkowski, P., Henning, T., Hinz, P. M., Hollis, J. M., Hyland, D. C., Kaltenegger, L., Labadie, L., Lane, B. F., Laughlin, G., Léger, A., Liseau, R., Mawet, D., Mennesson, B., Monnier, J. D., Quillen, A. C., Röttgering, H. J. A., Selsis, F., Serabyn, E., Wilner, D. J., Woolf, N. J., Traub, W. A., Gappinger, R. O., Ksendzov, A., Peters, R. D., and Scharf, D. P., "Terrestrial Planet Finder Interferometer (TPF-I) Whitepaper for the AAAC Exoplanet Task Force” (2007).

[11]Kong, E. M. C., Kwon, D. W., Schweighart, S. A., Elias, L. M., Sedwick, R. J., and Miller, D. W., "Electromagnetic formation flight for multisatellite arrays," Journal of Spacecraft and Rockets 41 (4), 659-666 (2004).

[12] Labeyrie, A., "Standing wave and pellicle: A possible approach to very large space telescopes," Astronomy and Astrophysics 77, L1-L2 (1979).

[13] Labeyrie, A., Guillon, M., and Fournier, J.-M., "Optics of 'laser trapped mirrors' for large telescopes and hypertelescopes in space,” Proc. SPIE 5899, 307-316 (2005).

[14] Kordyuk, A., "Magnetic levitation for hard superconductors,” Journal of Applied Physics 83 (1), 610-612 (1998).

[15] Shoer, J. P. and Peck, M. A., "Flux-pinned interfaces for the assembly, manipulation, and reconfiguration of modular space systems," The Journal of the Astronautical Sciences 57 (3), 667-688 (2009).

[16] Shoer, J., Wilson, W., Jones, L., Knobel, M., and Peck, M., "Microgravity demonstrations of flux pinning for station-keeping and reconfiguration of CubeSat-sized spacecraft,” Journal of Spacecraft and Rockets 47 (6), 10661069 (2010).

[17] Johansen, T. H. and Bratsberg, H., "Theory for lateral stability and magnetic stiffness in a high-Tc superconductormagnet levitation system,” Journal of Applied Physics 74 (6), 4060-4065 (1993).

[18]Bely, P. Y. (ed.), [The Design and Construction of Large Optical Telescopes], Springer-Verlag, New York, 260-261 (2003).

[19]Correlated Magnetics Research, "Coded Magnetic Structures and the Shortest Path Effect," http://www.correlatedmagnetics.com 


\section{Cornell University}

\section{A Flux-Pinning Mechanism for Segment Assembly and Alignment}

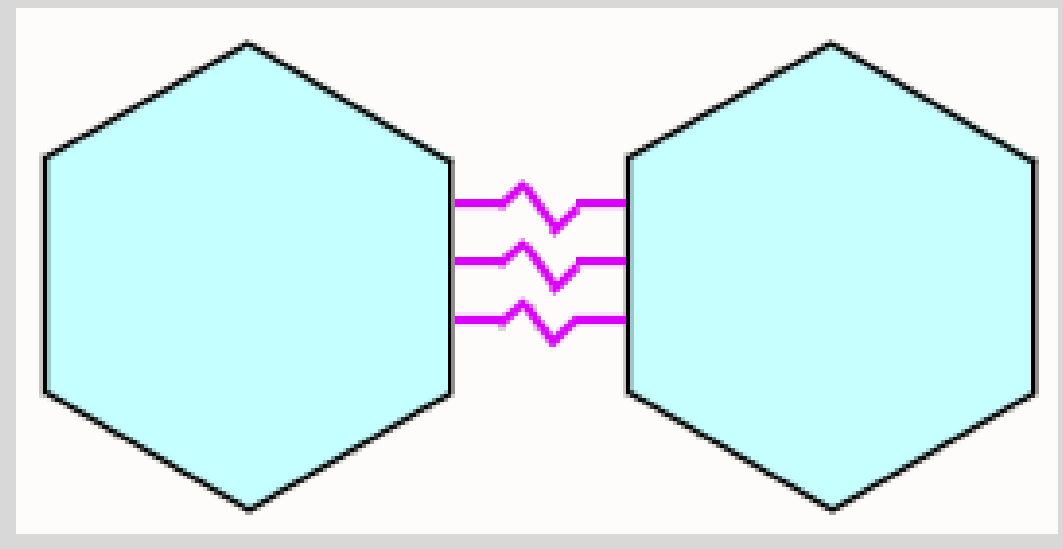

Jessica A. Gersh-Range

William R. Arnold

Mason A. Peck

H. Phillip Stahl 


\section{Introduction}

\section{Introduction}

Flux Pinning

The Mechanism

Assembly

Summary

\section{Current astrophysics questions include:}

- How did the first stars form?

-How did planets form?

-Are there large organic molecules in protostellar disks?

Answering these questions will require a

10- to $16-\mathrm{m}$ class far-infrared to

submillimeter space telescope. 


\section{Introduction}

\section{How to assemble a $10-$ to 16 -m mirror?}

\section{Introduction}

Flux Pinning

The Mechanism

Assembly

Summary
On-Orbit Deployment -JWST

- Tethers
On-Orbit Assembly

-Formation flight

-Electromagnetic formation flight - Laser trapping 


\section{Flux Pinning}

-A non-contacting interaction between a magnet and a type II superconductor

Flux Pinning

The Mechanism

Assembly

Summary

-Passively stabilizes the relative orientation and position in every direction that has a magnetic field gradient 


\section{Flux Pinning}

\section{Introduction}

Flux Pinning

The Mechanism

Assembly

Summary $\square$ Permanent Magnet

$\square$ Mobile Image

$\square$ Frozen Image

$\square$ Superconductor Surface

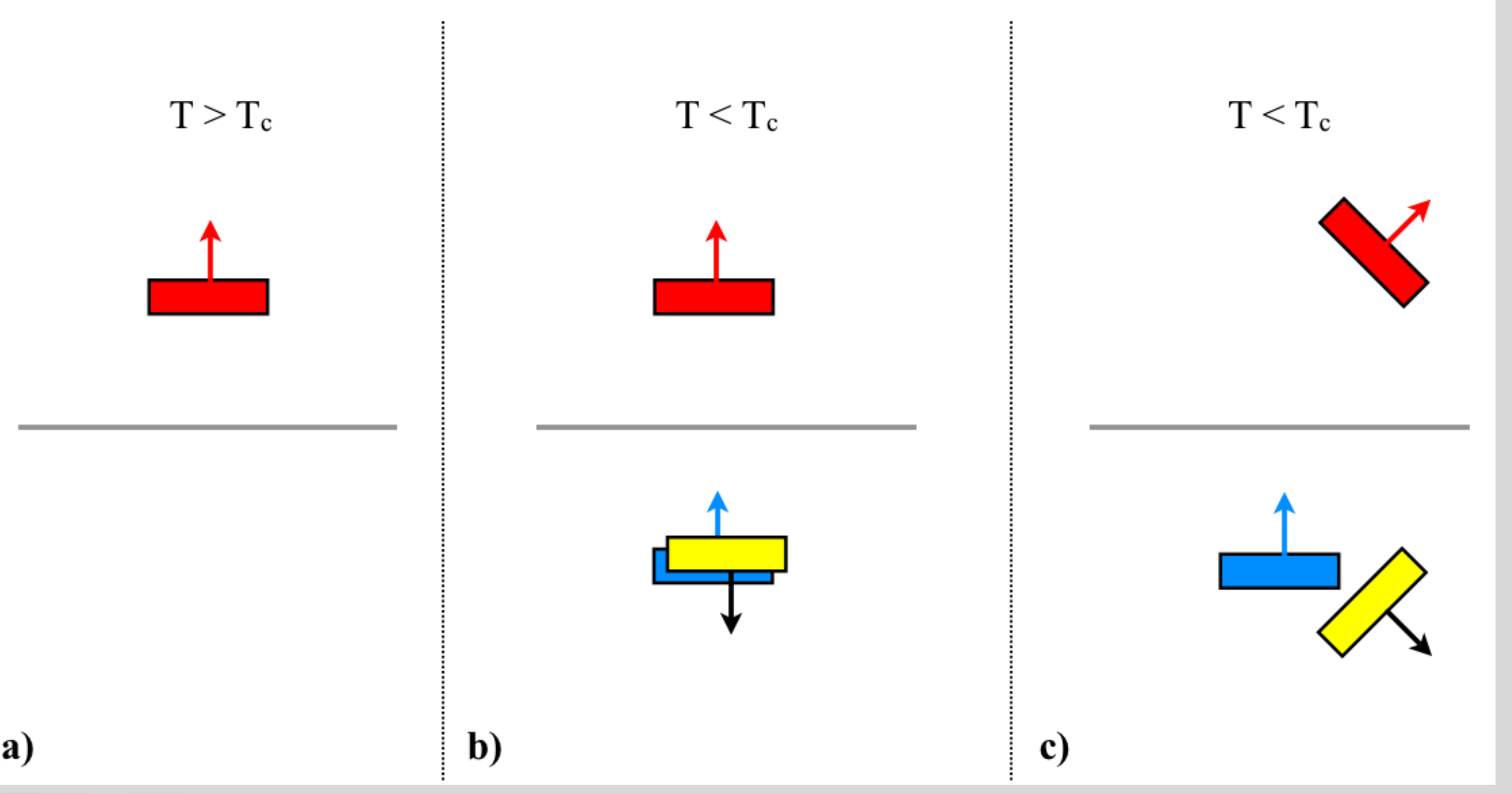




\section{The Flux-Pinning Mechanism}

Introduction

Flux Pinning

The Mechanism

Assembly

Summary
- Consists of at least one magnet and one superconductor

-Preferentially allows motion in specific degrees of freedom

-These degrees of freedom affect how the mechanism is constructed. 


\section{The Flux-Pinning Mechanism}

\section{Introduction}

Flux Pinning

The Mechanism

Assembly

Summary
Wire Flexure

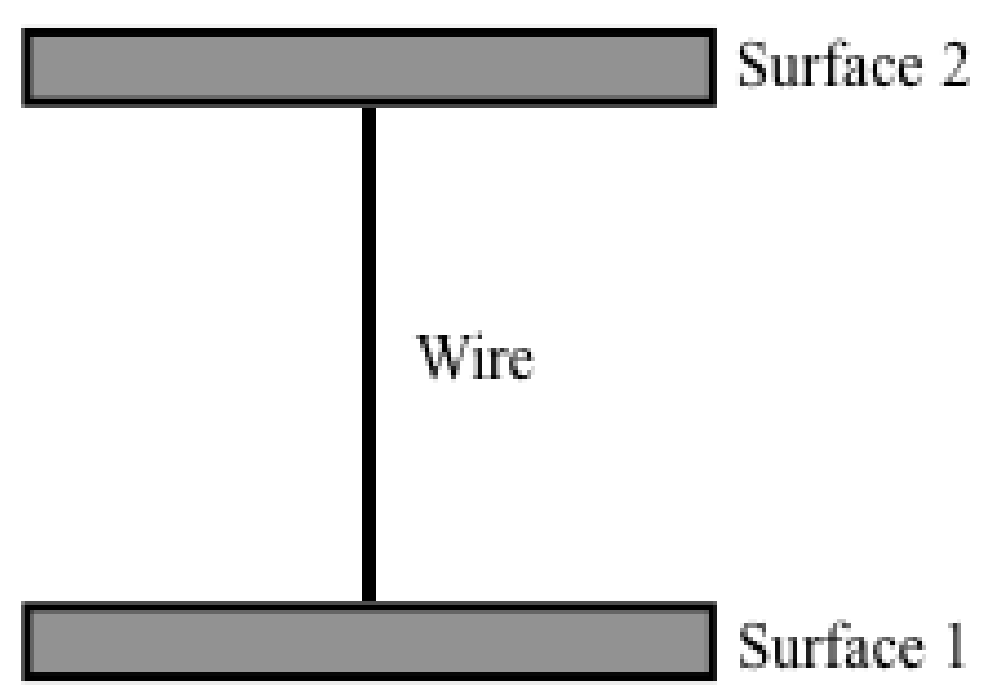

Analogous Flux-Pinning Mechanism
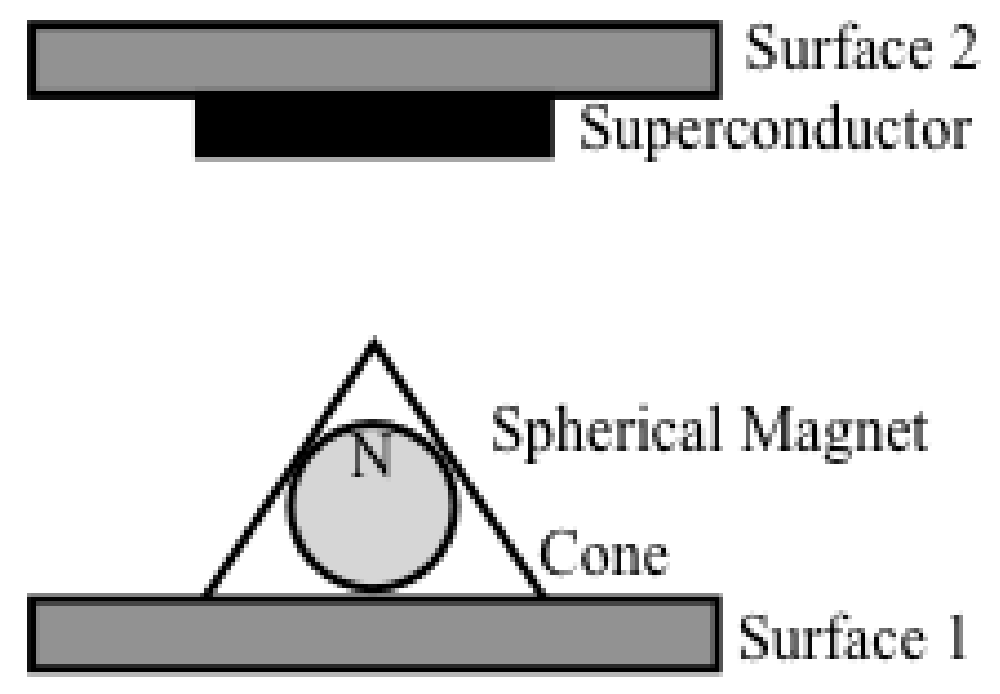


\section{The Flux-Pinning Mechanism}

\section{Introduction}

Flux Pinning

The Mechanism

\section{Assembly}

Summary
Parallel Blade Flexures

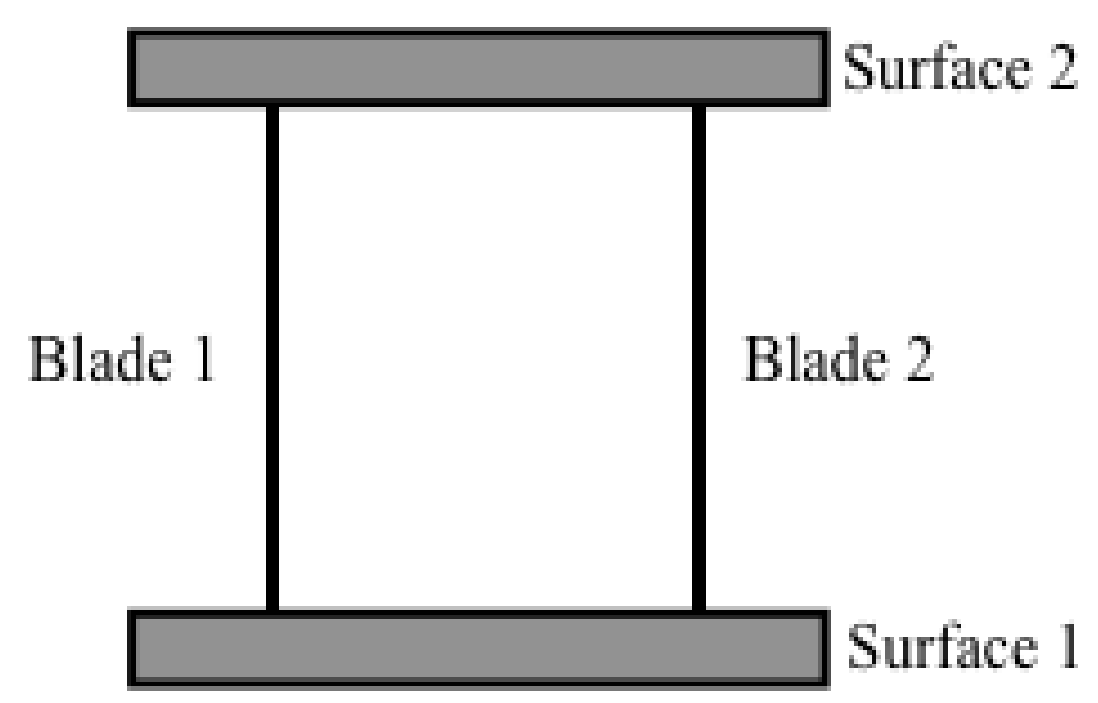

Analogous Flux-Pinning Mechanism

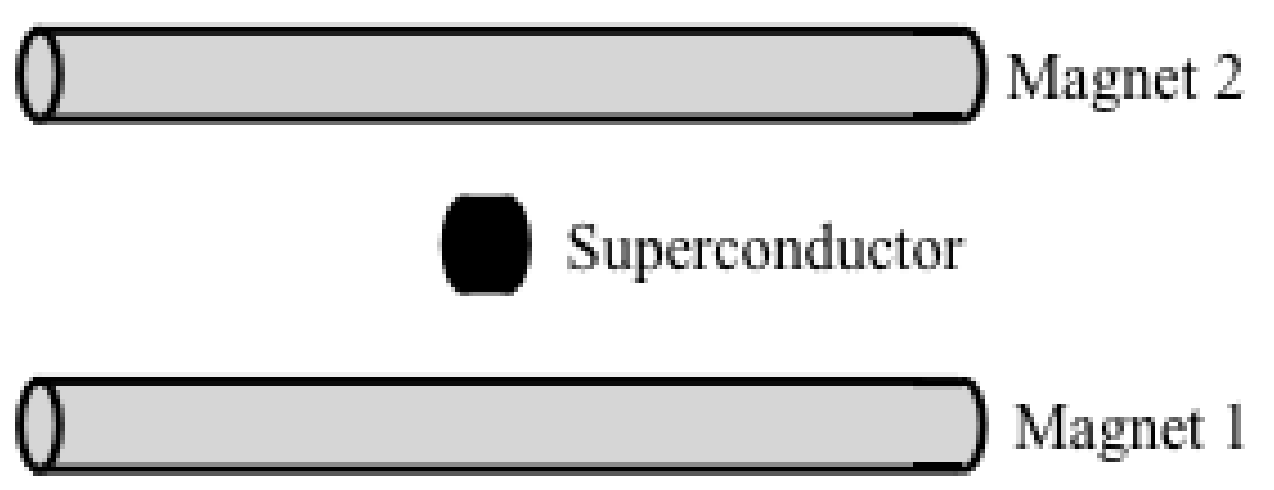




\section{The Flux-Pinning Mechanism}

Introduction

Flux Pinning

The Mechanism

Assembly

Summary
Cross-blade Flexure

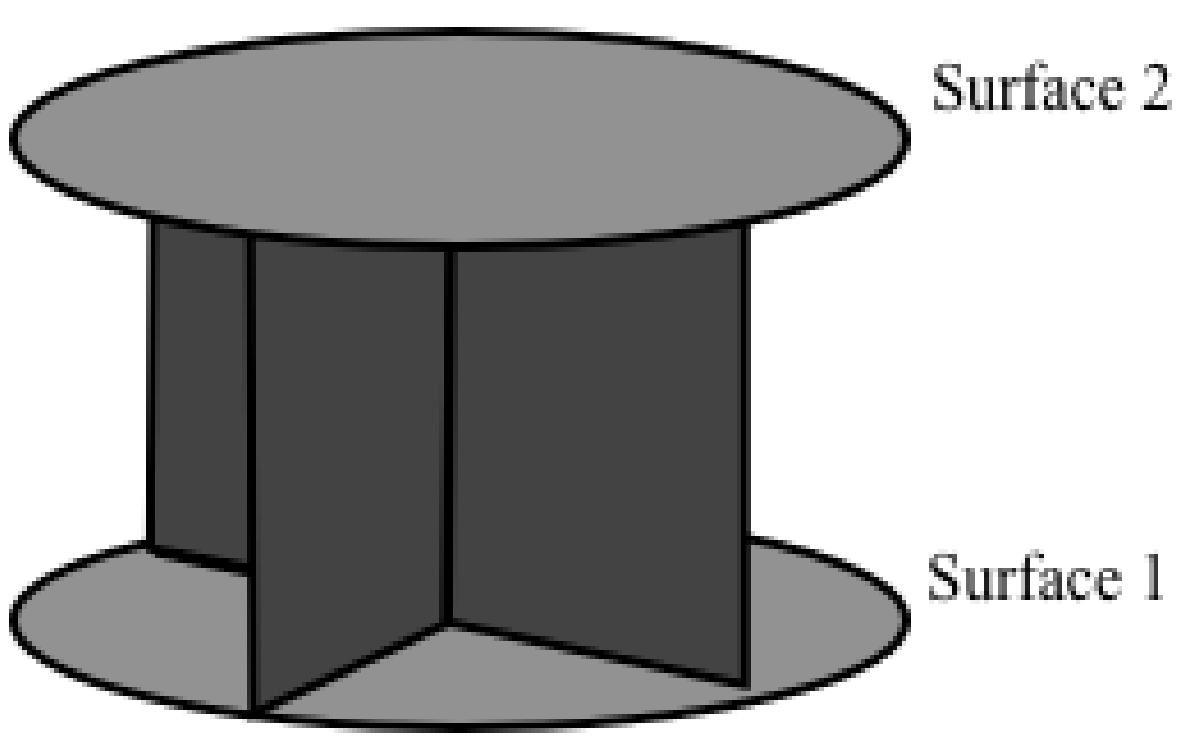

Analogous Flux-Pinning Mechanism
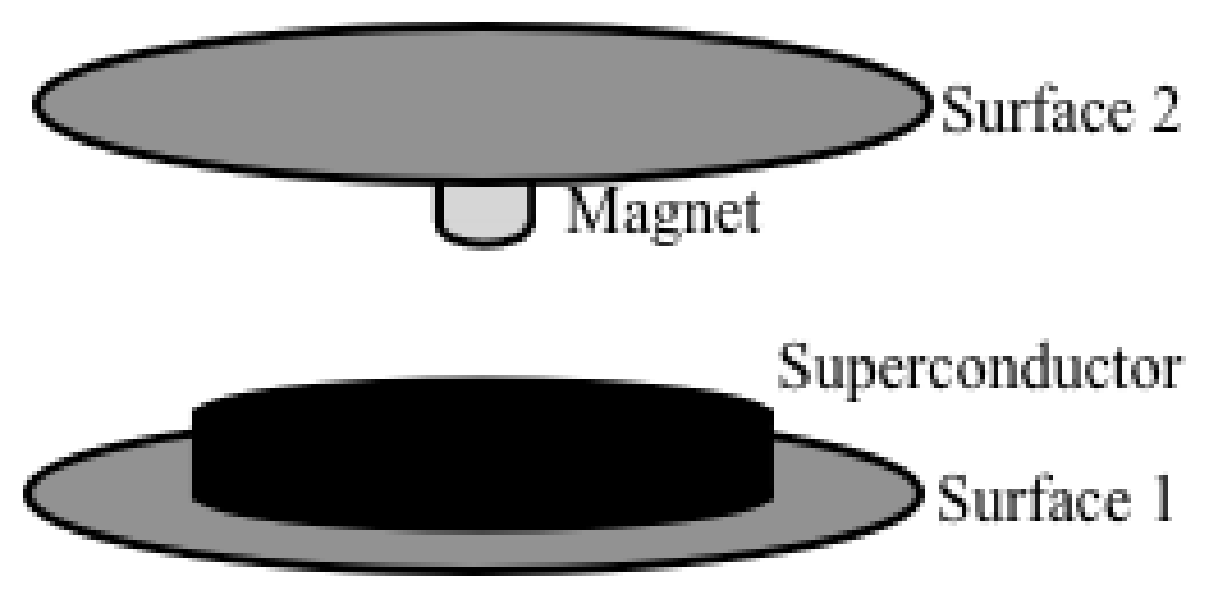


\section{The Flux-Pinning Mechanism}

Introduction

Flux Pinning

The Mechanism

Assembly

Summary

-The restoring force is analogous to a damped spring force.

-The stiffness and damping depend upon:

-The choice of magnet and superconductor

-The initial separation between the magnet and superconductor

-The presence of nonmagnetic conductors (such as aluminum) 


\section{Segmented Mirror Application}

Introduction

Flux Pinning

The Mechanism

\section{Assembly}

Summary

-Edges of neighboring mirror segments are connected by flux-pinning mechanisms

-Mechanisms add damping (adjustable by placing aluminum nearby)

-Mechanisms operate best at cryogenic temperatures

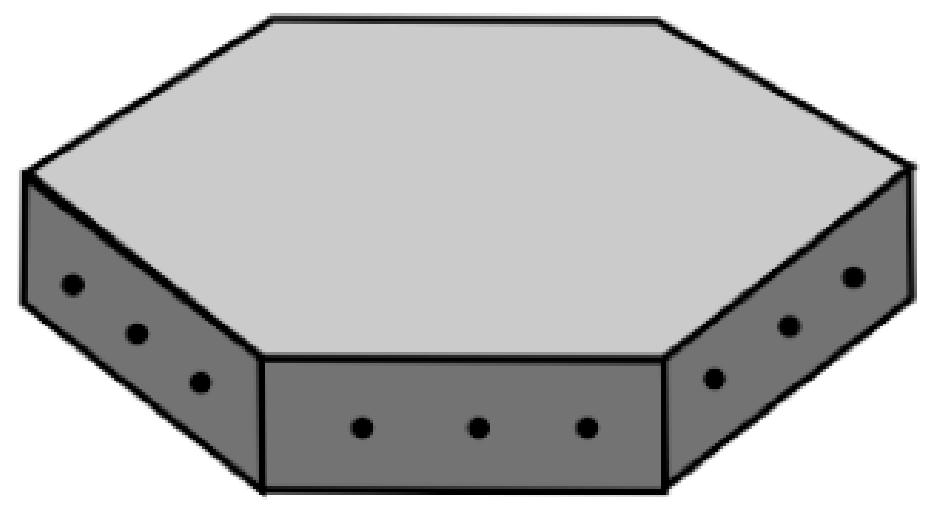

a)

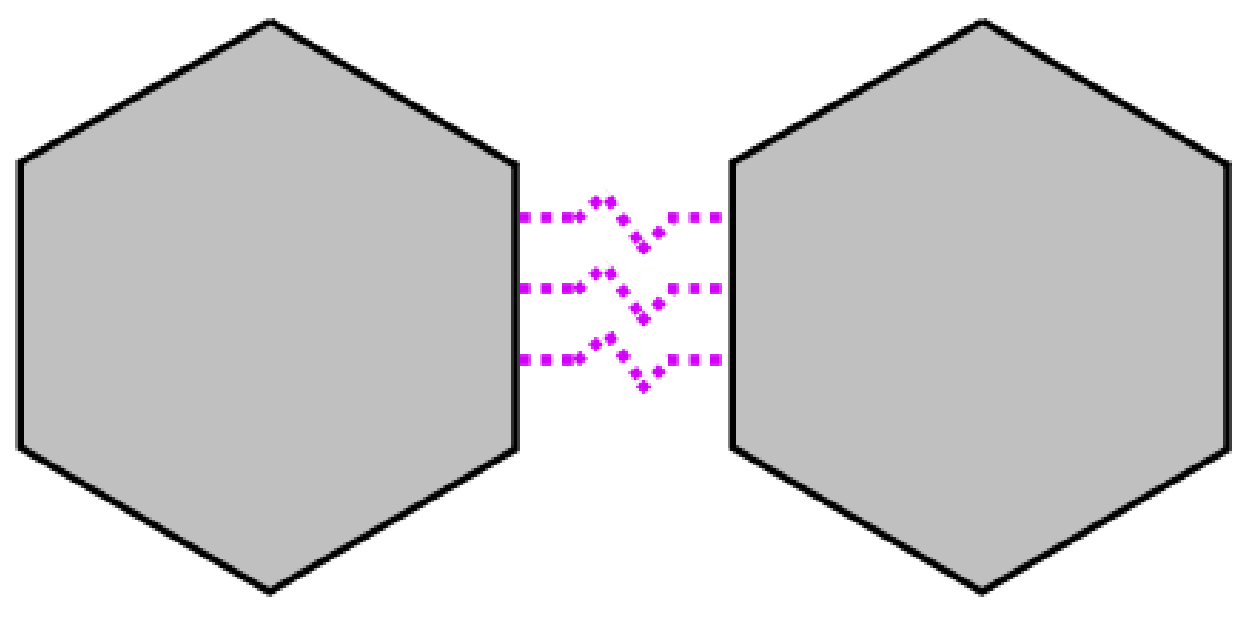

b) 


\section{Assembly Concepts}

Introduction

Flux Pinning

The Mechanism

\section{Assembly}

Summary
- Some other technique must be used to maintain the alignment as the superconductors cool.

-Possibilities include:

-Robotic Alignment

-Retractable Latches

-Temporary Active Control 


\section{Assembly Concepts}

Introduction

Flux Pinning

The Mechanism

\section{Assembly}

Summary
-Robotic Alignment:

-Robotic arms coarsely align the mechanisms.

-Other actuators (such as hexapods) finely align the mechanisms.

-The flux-pinning mechanisms cool below their critical temperature, and the other mechanisms are powered down.

Variant: Electromagnets provide the fine alignment. 


\section{Assembly Concepts}

Introduction

Flux Pinning

The Mechanism

\section{Assembly}

Summary
-Retractable Latches:

-Warm mirror assembled by latching the segments together.

-The latches release after the flux-pinning mechanisms cool.

Advantage: Can maintain the alignment without using any power in the event that the mirror must be warmed. 


\section{Assembly Concepts}

Introduction

Flux Pinning

The Mechanism

\section{Assembly}

Summary
-Temporary Active Control:

-Electromagnetic formation flight maintains the alignment as the superconductors cool.

-Electromagnets are placed far enough away from the mechanisms to prevent any interference.

-Electromagnets are turned off once the flux-pinning mechanisms cool. 


\section{Summary}

Introduction

Flux Pinning

The Mechanism

Assembly

Summary
-Flux-pinning mechanisms consist of magnets and superconductors.

-Motion is allowed in specific degrees of freedom.

-Motion in any other degree of freedom gives rise to a restoring force.

-The mechanism properties can be varied independently.

-Flux-pinning mechanisms require cryogenic temperatures to operate.

-Measurements of the achievable stiffness and damping are needed (in progress). 